\title{
A Comparison of Functional Fitness and Walking Speed with Cognitive Function of Facility-Dwelling Elderly Women: a Cross- Sectional Study
}

\author{
YONGNAM PARK ${ }^{1)}$, YOUNGSOOK BAE ${ }^{2)}$ \\ ${ }^{1)}$ Hu Nae Li Therapeutic Education Center \\ 2) Department of Physical Therapy, Daewon University College: 599 Shinwol-dong Jacheon-si, Chungbuk \\ 390-702, South Korea.TEL: +82 33-563-1103,E-mail:mecca87@hanmail.net
}

\begin{abstract}
Purpose] The purpose of this study was to compare functional fitness and walking speed with cognitive function of facility-dwelling older woman. [Subject and Methods] The subjects were 273 female residents of an elderly housing facility, aged 65 to 96 years old. The participants' cognitive function, walking speed and functional fitness were measured. The cognitive functions were measured with the MMSE questionnaire and walking speed was assessed by the 10 meter-walking time. The Senior Functional Fitness Test (SFFT) was used to measure upper and lower strength, aerobic endurance, upper and lower flexibility, agility and dynamic balance. The Senior Function Fitness Test includes six assessments: 30-Second Chair-to-Stand, Arm Curl, Chair Sit-and-Reach, Back Scratch, 8 Foot Up-and-Go, and Minute Step. [Results] The severe (decreased of cognitive function) group showed a significant decrease in all the items of functional fitness measured relative to the normal group, except for upper flexibility. Walking speed indicated significantly reduced cognitive function, and showed very significant correlations with upper and lower limb strength, aerobic endurance and agility. [Conclusion] This study showed that cognitive function is correlated to functional fitness in elderly women. Particularly, cognitive function is a factor that influences the reduction in muscle strength, flexibility, and endurance of the lower extremities. This indicates that reduced cognition function has a negative effect on walking speed and functional fitness of facility-dwelling elderly women.
\end{abstract}

Key words: Cognitive function, Functional fitness, Elderly women

(This article was submitted Jul. 21, 2011, and was accepted Sep. 8, 2011)

\section{INTRODUCTION}

Everybody goes through the aging process and this process, triggers unavoidable degradation in the physical, mental and social aspects of a person's capabilities and adaptability. The time period when people have difficulty in performing social functions due to such degradation is called the aged stage, and those in this stage are referred to as the elderly ${ }^{1)}$

As part of the aging process, older people experience impairment in physical function and increasing incidence of cognitive function problems. Physical strength means having the functional capacity or having the physical ability to perform activities on one's own without needing the help of others. Physical strength directly represents elderly persons' basic health condition as well as their ability to independently complete daily routines ${ }^{2,3)}$. Measures of functional capacity of the elderly walking speed and the Senior Functional Fitness Test (SFFT), which was developed by Jones and Rikli in 2002 ${ }^{2)}$. Walking speed is the best physical performance measure for predicting an elderly person's functional capacity ${ }^{4,5}$.

Cognitive functions enable the understanding and processing of information, recevied from the external environment and, by providing orientation regarding time and location, help individuals to organize complex information, comprehend what is happening in their daily lives, and judge and determine their condition. Thus, cognitive functions allow us to adapt to the environment around $u^{6,7)}$. Such cognitive functions gradually diminish as the aging process unfolds, and along with the malfunctioning of the elderly persons' central nervous systems, leads to difficulties in leading a normal, ordinary life or carrying out social activities ${ }^{6}$. The Mini Mental State Examination (MMSE) developed by Folstein et al. in 1975 is a popular method of measuring the cognitive function of elderly people.

The purpose of this study was to identify the relationship between functional fitness and walking speed and decrease of cognitive function of elderly women living in a facility.

\section{SUBJECTS AND METHODS}

\section{Subjects}

The participants in this study were 273 elderly women, aged 65 years and older (range 65 to 96 years old), who 
Table 1. Characteristics of subjects

\begin{tabular}{lcccc}
\hline & Severe $(\mathrm{n}=108)$ & Mild $(\mathrm{n}=82)$ & Normal $(\mathrm{n}=83)$ & All subjects $(\mathrm{n}=273)$ \\
\hline Age $($ year $)$ & $81.7 \pm 7.0$ & $77.8 \pm 7.6$ & $76.9 \pm 8.6$ & $79.4 \pm 7.9$ \\
Height $(\mathrm{cm})$ & $150.8 \pm 8.4$ & $153.2 \pm 9.5$ & $152.5 \pm 8.9$ & $152.1 \pm 8.8$ \\
Weight $(\mathrm{kg})$ & $51.0 \pm 8.9$ & $53.5 \pm 9.8$ & $56.7 \pm 117$ & $53.2 \pm 10.1$ \\
MMSE score & $11.6 \pm 2.0$ & $20.1 \pm 1.8$ & $26.5 \pm 1.8$ & $17.9 \pm 6.9$ \\
\hline
\end{tabular}

Values are presented as the mean \pm SD, Severe: MMSE score $<17$ point, Mild: MMSE score is $23-17$
point, Normal: MMSE score $>24$ point.

lived in a facility. The subjects understood that the purpose of the questionnaire was to measure their cognitive function, and they were all able to answer the questionnaire completely. Every participant was able to complete all the activities of functional fitness test and walk independently. After the MMSE measurements, subjects were divided into three cognitive function groups (severe decrease, mild decrease and normal groups) according their MMSE scores. Then the SFTT scores and walking speeds of the three groups were compared. Table 1 illustrates the subject characteristics, the assessment results, and MMSE scores.

\section{Method}

Weight and height were measured for all subjects. Then, each subject completed the MMSE questionnaire, the Senior Function Fitness Test and the walking speed assessment.

Cognitive function was measured using the Mini Mental State Examination screening tool. The MMSE has questions in six sections, which represent different cognitive functions ${ }^{8)}$. The maximum score is 30 points and a score of 24 points or higher shows that cognitive function is normal. A score of 23 points or less suggests evidence of cognitive impairment; a score between 18 and 23 points indicates mild impairment, and a score of 17 or less indicates severe impairment ${ }^{9,10)}$.

The Senior Functional Fitness Test evaluates a person's functional fitness defined as the physiologic capacity to perform normal everyday activities safely and independent without undue fatigue ${ }^{11)}$, and consists of six physical fitness measurements:

(1) The 30-Second Chair-to-Stand test that assesses lower body strength. The score is the number of times in 30 seconds a subject can stand up completely from a chair with arms folded across the chest.

(2) Arm Curl test assesses upper body strength. The score is the number of biceps curls that can be completed in 30 seconds holding a hand weight ( $2.27 \mathrm{~kg}$ for women).

(3) The Chair Sit-and-Reach test assesses lower body flexibility. The score is the number of $\mathrm{cm}(+$ or -$)$ between extended middle fingers and tip of the toes, from a sitting position at the front of a chair with one leg extended and hands reaching toward the toes.

(4) The Back Scratch test assesses upper body flexibility. The score is the number of $\mathrm{cm}(+$ or - ) between extended middle fingers, with one hand reaching over the shoulder and the other up the middle of the back.

(5) The 8 Foot Up-and-Go test assesses agility/dynamic balance. The score is calculated as the number of seconds required to get up from a seated position, walk 8 feet $(2.44$ $\mathrm{m})$, turn and return to the seated position.

(6) The 2-Minute Step test assesses aerobic endurance. The score is the number of times in two minutes that the subjects can alternately raise each knee to a point midway between the patella and the iliac crest.

The walking speed test is an easy assessment tool that is a useful indicator of activities of daily living (ADL) and physical function of elderly people $\mathrm{e}^{12,13)}$ and it is the preferred tool for measuring the physical fitness of community-dwelling elderly ${ }^{13}$. Walking speed is assessed by having the subjects walk 10 meters distance. The test was completed three times and the scores were averaged.

\section{Statistics}

Statistical analyses were performed using SPSS version 12 for Window (SPSS Institute Korea, Seoul, Korea) and variables are presented as Mean $\pm \mathrm{SD}$.

ANOVA was used for comparison of the six items of SFFT and walking speed, and multiple comparison analyses of the three groups were used Least-Significant-Difference (LSD) test. Pearson's correlation coefficients were used to calculate the correlations between the MMSE score, the walking speed and the SFFT score. Statistical significance was chosen as $\mathrm{p}<0.05$.

\section{RESULTS}

The characteristics of 273 participants in this study are shown Table 1. Cognitive function decreases in the elderly, and differences between walking speed and SFFT measurement are shown in Table 2 . Walking speed was very significantly associated with a reduction in cognitive function $(\mathrm{p}<0.01)$. For five tests in the SFFT (30 Second Chair-to-Stand, The Arm Curl, The Chair Sit-to-Reach, The 2 Minute Step, and the 8 Foot Up-and-Go), subjects in the normal group performed better than the severe group $(p<0.05$ and $p<0.01)$. The distance in the Back Scratch test of the SFFT did not differ significantly between the groups. Functional fitness, as indicated by the SFFT score and walking speed, showed very significant correlations with the 30 Second Chair-to-Stand, the Arm Curl, the 2 Minute Step and the 8 Foot Up-and-Go test (Table 3).

\section{DISCUSSION}

The aim of this study was to identify the correlation between functional fitness and walking speed and the cognitive function decreases in elderly women who dwelled 
Table 2. Comparison of SFTT items and walking speed by MMSE score

\begin{tabular}{lccr}
\hline & Severe $(\mathrm{n}=108)$ & \multicolumn{1}{c}{ Mild $(\mathrm{n}=82)$} & \multicolumn{1}{c}{ Normal $(\mathrm{n}=83)$} \\
\hline Walking speed (sec/m) & $2.14 \pm 0.93^{* *}$ & $1.46 \pm 0.61^{*}$ & $1.38 \pm 0.43$ \\
Senior physical fitness test & & & \\
30-second Chair-to-stand (time) & $5.19 \pm 2.88^{* * \dagger}$ & $8.71 \pm 3.54^{*}$ & $9.43 \pm 2.84$ \\
Arm curl (time/min) & $12.24 \pm 6.17^{* \dagger}$ & $15.13 \pm 8.51$ & $17.71 \pm 5.62$ \\
Chair sit-and-reach (cm) & $5.10 \pm 1.08^{* *}$ & $4.84 \pm 2.22$ & $3.40 \pm 1.95$ \\
Back scratch (cm) & $13.45 \pm 7.18$ & $14.25 \pm 5.22$ & $10.72 \pm 2.20$ \\
2-min step (time) & $31.31 \pm 7.48^{* *}$ & $55.38 \pm 5.03$ & $56.75 \pm 4.13$ \\
8 foot up-and-go (sec) & $5.94 \pm 2.32^{*}$ & $4.27 \pm 2.27$ & $4.62 \pm 2.11$ \\
\hline
\end{tabular}

${ }^{*} \mathrm{p}<0.05$ compared to the value in the normal group; ${ }^{* *} \mathrm{p}<0.01$ compared to the value in the normal group; ${ }^{\dagger} \mathrm{p}<0.05$ compared to the value in the mild group; ${ }^{\dagger \dagger} \mathrm{p}<0.01$ compared to the value in the mild group by ANOVA test and LSD test.

in a facility.

Cognitive functions decrease as part of the normal aging process and this affects the activity levels of the elderly ${ }^{14}$. According to the results of this study, the severe group showed a significant decrease in all the measured items relative to the normal group $(\mathrm{p}<0.05)$, except for the Back Scratch test. This means that the degradation of cognitive functions triggers a reduction in active physical strength. In particular, two groups (the normal group and the severe group) showed very significant differences in three items; the 30-Second Chair-to-Stand, the Chair Sit-and-Reach, and 2-minute Step. Therefore, it can be assumed that a decrease in cognitive function is a factor that influences the reduction in muscle strength, flexibility, and endurance of the lower limbs. These physical factors increase the chance of falling ${ }^{15,16)}$ or slipping while getting in and out the bathtub or climbing stairs ${ }^{2}$. Other studies have shown that decreased cognitive function is related to loss of independence of ADL and social activity ${ }^{17,18)}$.

Walking speed is a factor that predicts elderly people's functional abilities ${ }^{19,20)}$. Hiroya and Mituyo ${ }^{13)}$ utilized gait speed to measure elderly people's physical functions, and the results of their study demonstrate that elderly women's walking speed changed significantly in accordance with a reduction in cognitive functions. In addition, walking speed had a very significant correlation with the lower and upper limb muscle strength, endurance, and levels of alertness. Functional fitness, the ability to perform normal everyday activities independently and safely, is one of the most important issues for older people ${ }^{21)}$.

As discussed above, the degradation of cognitive functions may cause problems in ADL and instrumental activities of daily living (IADL) that require adequate lower limb muscle strength, endurance and alertness. The degradation of active physical strength, which is related to ADL and IADL, may influence the lives of institutionalized elderly people. This indicates that reduced cognitive function has a negative effect on walking speed and functional fitness on facility-dwelling elderly women.

Cognitive function correlates with functional fitness in elderly women. Particularly, decreasing cognitive function is a factor that indicates reduction in muscle strength, flexibility and endurance of the lower limbs.
Table 3. Correlation of between the Walking speed with SFTT items scores

\begin{tabular}{cc}
\hline & Walking speed \\
\hline 30 -second Chair-to-stand & $-0.723^{* *}$ \\
Arm curl & $-0.627^{* *}$ \\
Chair sit-and-reach & $0.214^{*}$ \\
Back scratch & 0.116 \\
2 min step & $-0.510^{* *}$ \\
8 foot up-and-go & $0.580^{* *}$ \\
\hline
\end{tabular}

$* \mathrm{p}<0.05, * * \mathrm{p}<0.01$ by Pearson's correlation analysis.

Therefore, the environment of nursing homes should be properly modified to reflect elderly people's level of cognitive abilities. The cognitive performance could be accounted for by sex ${ }^{14)}$, but this study focused only on elderly women. The results of this study may only be generalized with care because the subjects included only elderly females. Therefore, additional research is necessary.

\section{REFERENCES}

1) Tanaka K, Wojtek J: Successful aging in the New Millenium: A Japanese Perspective. Bulletin of Institute of health and sport science university of Tsukuba, 1998, 21: 1-13.

2) Johns CJ, Rikli R: Measuring function fitness of older adult. J Active Aging, 2002: 24-30.

3) Kim HS, Tanaka K: The assessment functional age using "activities of daily living" performance tests: A study of Korea woman. J Aging and Physical Activity, 1995, 3: 39-53

4) Murry R, Huelslnetter MM, O'Doscroll D: Nursing process in later Maturity. Prentice Hall, 1980.

5) Najebson T, Rahmani K, Elazer B: An elementary cognitive assessment and treatment of carniocerebrally injured patient. New York: Plenum, 1984.

6) Carole BL: Aging: The health-care Challenge, 4th ed. Philadelphia: FA DAVIS, 2002.

7) Folstein MF, Folstein SF, McHugh PR: "Mini-mental state": a practical method for grading the cognitive state of patient for the dementia. J Psychiar Res, 1975, 12: 180-198.

8) Tonbaught TN, Mchutye NJ: The mini-mental state examination: A comprehensive review. J AM Geriatr Soc, 1992, 10: 922-935.

9) Crook T, Ferris S, Raymond B: Assessment in geriatric psychopharmacology, New Canaan, Conn, 1983. 
10) Rikli RE, Jones CJ: Development and validation of functional fitness tes for community -residing older adults. J Aging Phys Act, 1999, 12: 127-159.

11) Tainaka K, Aoki J: Fitness-related factor associated with changes in walking speed elderly woman. Jpn J Phys Fitness Sports Med, 1994, 43: 343-351.

12) Potter MM, Evans PL, Duncan M: Gait speed and activity daily of living function in geriatric patient. Archi of Physical and Medicine, 1995, 76 997-999.

13) Hiroya M, Mituyo N: The relation of walking velocity with motor ability and functional capacity in the community dwelling elderly. J Phys Ther Sci, 2008, 20: 59-62.

14) Christensen $\mathrm{H}$, Korten A, Jorm AF, et al.: Activity level and cognitive functioning in an elderly community sample. Aging Ageing, 1996, 25: 72-80.

15) Tinetti ME, Speechley M, Ginter S: Risk factors for falls among elderly persons living in the community. N Engl J Med,. 1988, 319: 1701-1707.
16) Kachthaler T: Fall in the institutitional elderly. J AM Geriatric, 1978, 26 : 424.

17) Tabbarah M, Crimmins EM, Seeman TE: The relationship between cognitive and physical performance: MacArthur Studies of Successful Aging. J Gerontol Med Sci, 2002, 57A: 228-235.

18) Moritz DJ, Kasl SV, Berkman LF: Cognitive functioning and the incidence of limitations in activities of daily living in an elderly community sample. Am J Epidemiol, 1995, 141: 41-49.

19) Koyano W, Shibata H, Nakazato K, et al.: Measurement of competence: reliability and validity of the TMIG Index of /conpentence, Arch agerontol Geriatr, 1991, 13: 103-116.

20) Potter JM, Evas AL, Duncan G.: Gait and activities of daily living function in geriatric patient. Arch Phys Med Rehabi, 1995,76: 997-999.

21) Bohannon RW, Eriksrud O: What measured of lower extremity muscle strength best explains walking independence. J Phys Ther Sci, 2001, 13: $1-3$. 